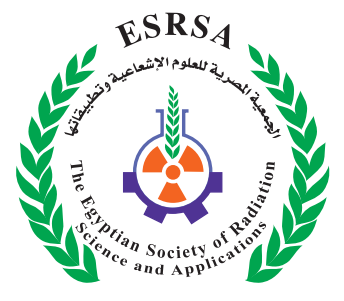

J. Nucl. Tech. Appl. Sci., Vol. 7, PP. 209 : 220 (2019)

\title{
A Study of Single Photon Emission Computed Tomography by using Geant4/GATE
}

Hussein, M.T. ${ }^{1}$; Ibrahim H.F. ${ }^{1}$; Morad E.A. ${ }^{1}$; Maamoun I.K. ${ }^{2}$; Attala E.M. ${ }^{3}$

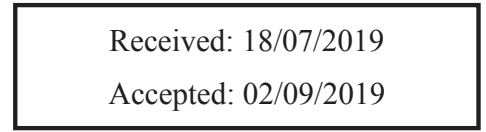

emanahmedmostafamorad@gmail.com

\section{KEYWORDS}

SPECT, Quality

Control, Quadrant

Bar Phantom, Monte

Carlo, GATE.

\section{ABSTRACT}

The aim of this research is to validate and investigate the Geant4/ GATE platform for the simulation of a dual-head detector SIEMENS Symbia E gamma camera installed in El-Kaser El-Ainy (Nuclear Medicine and Radiotherapy Unit) at Cairo University. Each camera consists of a lead collimator, scintillation crystal, and head shielding. The back compartment consists of photomultiplier tubes and electronic circuits behind the crystal. The photoelectric effect, Compton scattering, atomic ionization, bremsstrahlung, multiple scattering, and Rayleigh scattering are included in the simulated physics processes. The experimental data from the Quality Control measurements (Energy resolution, Uniformity, Spatial resolution and Sensitivity) versus the simulation results from GATE are compared.

All The measurements are performed by using the radioisotope Tc$99 \mathrm{~m}$ to determine the energy resolution and extrinsic sensitivity with a Low Energy High Resolution (LEHR) collimator and to ensure the quality of the scintillation crystal without collimator by measuring the uniformity. Other collimators are also used to estimate their extrinsic spatial resolutions and the corresponding extrinsic sensitivity values. In addition, a quadrant bar phantom consisting of 4 sets of lead bars in each quadrant is used to determine the Modulation Transfer Function (MTF) as a characteristic of the camera intrinsic spatial resolution.

1. Physics Department, Faculty of Science, Cairo Univeristy.

2. Medical Imaging Unit, Faculty of Medicine, Cairo University.

3. Radiation Oncology Department, National Cancer Institute, Cairo University. 


\section{ABBREVIATION:}

NM Nuclear Medicine, QC Quality Control, LEHR Low Energy High Resolution, LEGP Low Energy General Purpose, LEHS Low Energy High Sensitivity, MC Monte Carlo, QB Quadrant Bar, MTF Modulation Transfer Function.

\section{INTRODUCTION}

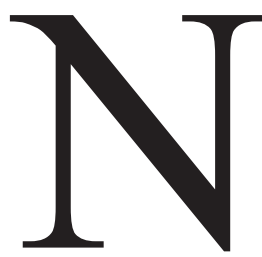

uclear Medicine (NM) provides noninvasive tools by using radioactive materials for the imaging, diagnosis and treatment of patients. An imaging system plus compatible radiopharmaceuticals are the two major ingredients of any imaging procedure in NM. Single Photon Computed Tomography (SPECT) and Positron Emission Tomography (PET) in NM depend on the creation of functional images and have the advantages of high intrinsic sensitivity as well as unlimited depth penetration.

SPECT is a modern rotating gamma camera which can produce $2 \mathrm{D}$ or $3 \mathrm{D}$ images representing the distribution of the injected or ingested radioactive tracers in the body such as Tc-99m, I-123, I-131, Ga-67, and Tl-201, but Tc-99m is the most commonly used radioisotope in SPECT imaging and diagnosis. SPECT can be single-head or multi-head system and currently is considered the most widely used emission tomography method due to the longer halflife and cost-effectiveness of its radio-tracers which produce single gamma photons with specific energies. The general components of any SPECT system include a collimator, a scintillation crystal coupled with an array of photomultiplier tubes (PMTs) and additional electronics for signal processing. The main purpose of SPECT is the imaging of tumors, tissue abnormalities and other internal deficiencies of the organs. In SPECT, "single photon" means that single photons are detected without any coincidence unlike the PET equipment. The observed spatial dis- tribution of the radioactivity concentration inside the body is used to diagnose the functions and anatomies of the patient in 2D or 3D (tomographic) images.

The procedures of Quality Control (QC) are very important for any SPECT system. They provide the confidence to the medical physicists and physicians that the resulting SPECT scan supplies an accurate representation of the radioisotope distribution in the patient (IAEA, 2009). Therefore, it is extremely necessary to calibrate the commissioned SPECT systems periodically to ensure the validity and accuracy of the resulting images. Energy resolution, spatial resolution, uniformity and sensitivity are among the main procedures of QC for any SPECT systems.

Generally speaking, a Monte Carlo (MC) method is a numerical solution to a physical problem that models objects interacting with each other or with their environment based on random number generators. The MC simulation techniques have been found to be very powerful tools for the studying of the available NM modalities, particularly for the QC and dosimetry procedures. It is also one of the most accurate computational techniques to design new medical imaging systems and to estimate the expected particle interactions within that system based on the statistical nature of photon emissions and particle interactions with matter.

The Geant4 Application for Tomographic Emission, GATE (Buvat et al., 2002; Jan et al., 2004) is a software package written in $\mathrm{C}++$ based on the Geometry and tracking version 4, Geant4, toolkit (Agostinelli et al., 2003) that can model and simulate different medical physics modalities such as SPECT, PET, Computed Tomography (CT) and radiotherapy (RT). Geant4 is a modern MC platform used for tracking particles, describing geometry, defining materials, optimizing physical processes and controlling detector responses among other aspects of the physical system. 


\section{MATERIALS AND METHODS}

\section{Experimental Setup}

In this study, all measurements were carried out by using a SIEMENS Symbia E SPECT (SIEMENS, 2014) installed in El-Kaser El-Ainy Hospital at Cairo University. The equipment and its head components are shown in Fig. 1. The basic specifications

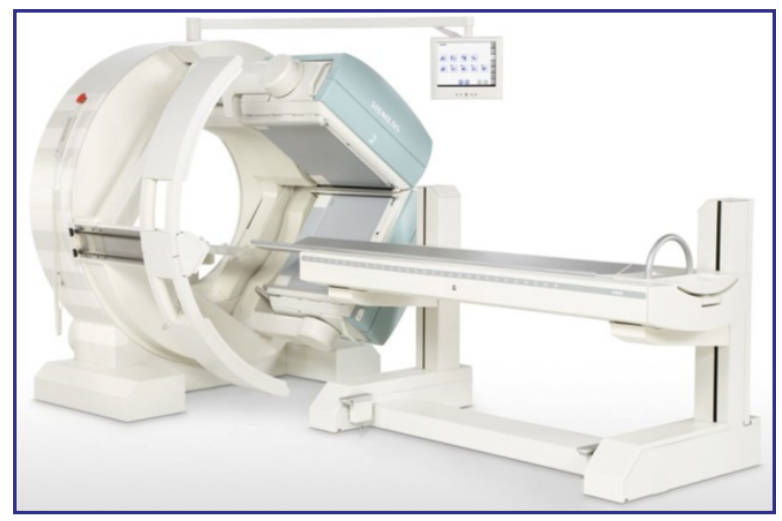

(a) Full system of the two cameras are listed in Table 1. It includes a removable Low Energy High Resolution (LEHR) lead collimator, Sodium Iodide doped with Thallium (NaI(TI)) scintillation crystal, a light guide and 59 photomultiplier tubes (PMTs). The LEHR collimator features parallel holes with hexagonal patterns and is used for low energy sources such as Tc-99m.

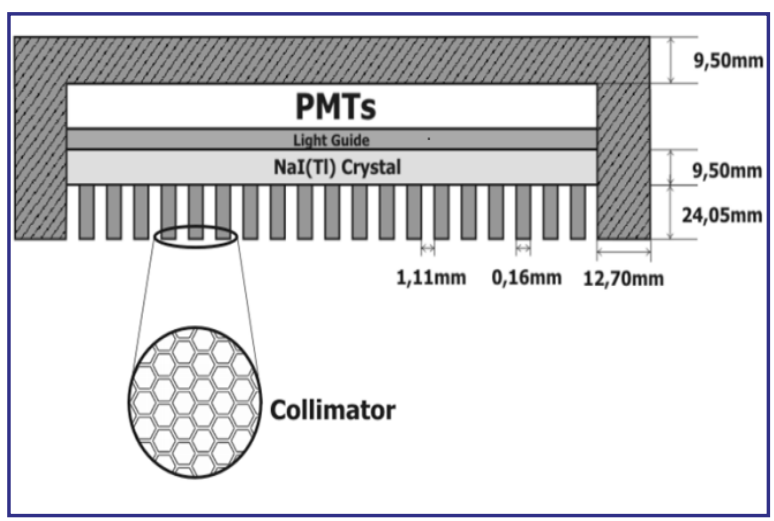

(b) Camera structure

Fig. (1): SIEMENS Symbia E.

The energy resolution of the gamma camera was measured extrinsically with a Tc-99m point source activity of $300 \mu \mathrm{Ci}$ at $25 \mathrm{~cm}$ from the surface of the collimator. The intrinsic uniformity of the SPECT system was determined at a distance of $210 \mathrm{~cm}$ between the crystal surface and the $300 \mu \mathrm{Ci}$ Tc-99m source. The intrinsic spatial resolution was evaluated by using a Quadrant Bar (QB) phantom with

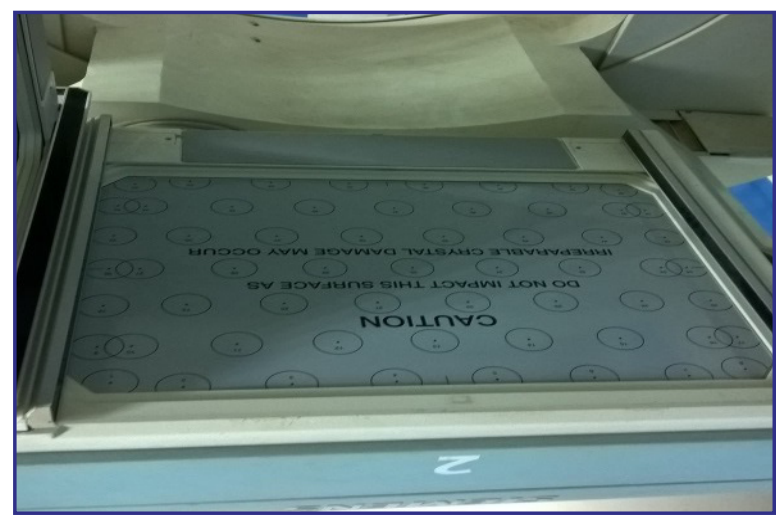

(a) bar widths of $9.525,6.35,4.763$, and $2.54 \mathrm{~mm}$ placed on top of the scintillation crystal by using a $300 \mu \mathrm{Ci}$ Tc- $99 \mathrm{~m}$ source activity located at $235 \mathrm{~cm}$ from the phantom surface and then rotating the phantom by $90^{\circ}$ repeatedly to take four different images. Fig. 2 shows the camera crystal without and with the QB phantom on top, after removing the collimator.

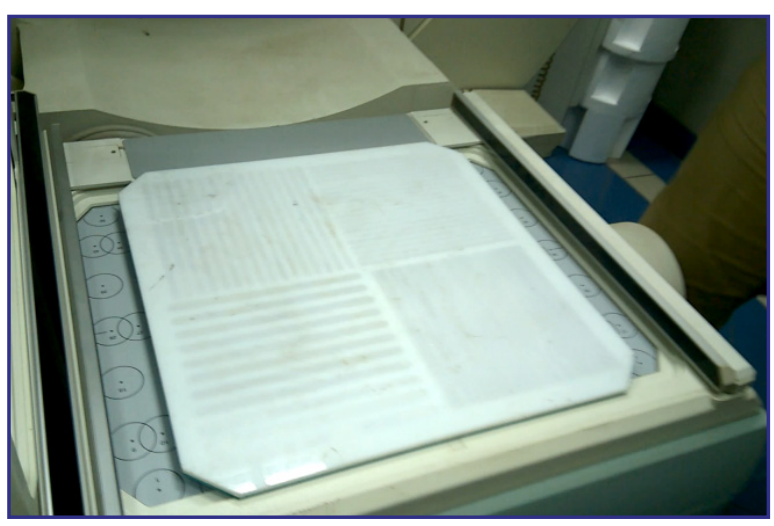

(b)

Fig. (2): Symbia E camera crystal without (a) and with (b) the QB phantom. 
Table (1) : Specifications of Symbia E.

\begin{tabular}{|c|c|c|}
\hline \multirow{2}{*}{$\begin{array}{c}\text { Detector } \\
\text { Dimensions }\end{array}$} & FFOV & $53.3 \times 38.7 \mathrm{~cm}^{2}$ \\
\hline & Diagonal FFOV & $65.9 \mathrm{~cm}$ \\
\hline \multirow{3}{*}{ Crystal } & Type & $\mathrm{NaI}(\mathrm{Tl})$ \\
\hline & Size & $59.1 \times 44.5 \mathrm{~cm}^{2}$ \\
\hline & Thickness & $9.5 \mathrm{~mm}$ \\
\hline \multirow{2}{*}{$\begin{array}{l}\text { Detector } \\
\text { Shielding }\end{array}$} & Back & $9.5 \mathrm{~mm}$ \\
\hline & Side & $12.7 \mathrm{~mm}$ \\
\hline \multirow{4}{*}{ LEHR } & No. of holes \& Shape & $148,000 \&$ Hexagonal \\
\hline & Hole Length & $24.5 \mathrm{~mm}$ \\
\hline & Septal diameter & $0.16 \mathrm{~mm}$ \\
\hline & Hole diameter & $1.11 \mathrm{~mm}$ \\
\hline \multirow{4}{*}{ LEGP } & No. of holes \& Shape & $90,000 \&$ Hexagonal \\
\hline & Hole Length & $24.5 \mathrm{~mm}$ \\
\hline & Septal diameter & $0.2 \mathrm{~mm}$ \\
\hline & Hole diameter & $1.45 \mathrm{~mm}$ \\
\hline \multirow{4}{*}{ LEHS } & No. of holes \& Shape & $28,000 \&$ Hexagonal \\
\hline & Hole Length & $24.5 \mathrm{~mm}$ \\
\hline & Septal diameter & $0.36 \mathrm{~mm}$ \\
\hline & Hole diameter & $2.54 \mathrm{~mm}$ \\
\hline
\end{tabular}

\section{Simulation Models}

The dual head SPECT was modeled by MC simulations in GATE to compare the gamma camera measured data taken during the QC procedures or calculated offline with the simulation results (Park et al., 2009; Momennezhad et al., 2012; Taherparvar et al., 2018). Two categories of suitable electromagnetic physical processes can be included in the GATE simulation: Standard-energy electromagnetic (EM) processes and low-energy EM processes (Zaidi, 1999). The simulations were done by using the standard EM model because it is much faster and more efficient in computation than the other EM models. The processes of this model include the photoelectric effect, Compton scattering, atomic ionization, bremsstrahlung, multiple scattering, and Rayleigh scattering. In order to perform the simulation in GATE accurately, the important parts of the system had to be defined precisely. Energy cuts were applied on all the simulated models except for the energy resolution QC to obtain the full energy spectrum.

\section{Dual-Head Gamma Camera}

All the used simulation models include the photon tracking through the dual-head of the SPECT system. Material and geometrical characteristics such as the composition and thickness of the crystal and the collimator were implemented according to the technical specifications of Symbia E. The base of the model structure was represented by the "world" volume. The "world" volume represents a box centered at the origin with size large enough to include the whole simulation geometry. When any particle escapes from the world volume, the tracking of this particle stops. The world contains other sub- 
volumes referred to as daughter volumes and every daughter volume has specific purpose and name. Fig. 3 illustrates the GATE visualization of the dual-head

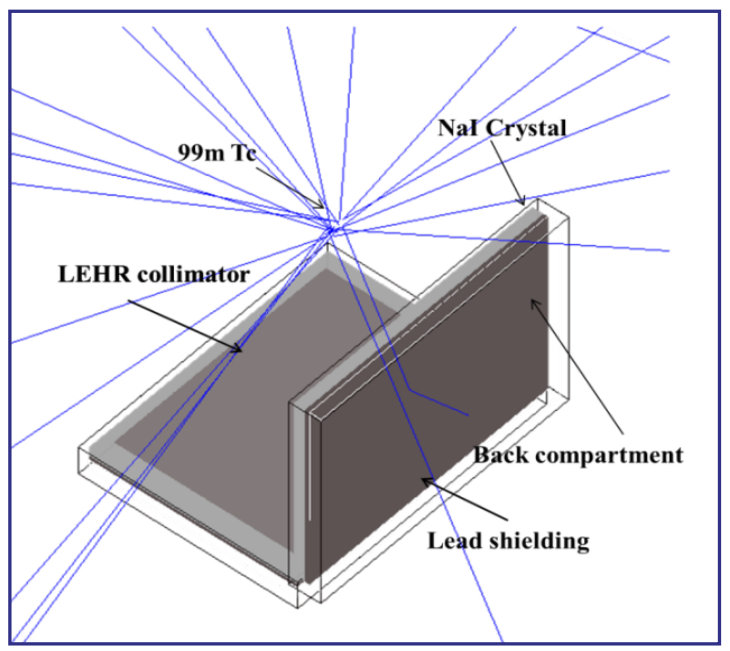

(a) Dual-head cameras gamma cameras of the Symbia E including the hexagonal collimator, scintillation crystal, back compartment and lead shielding.

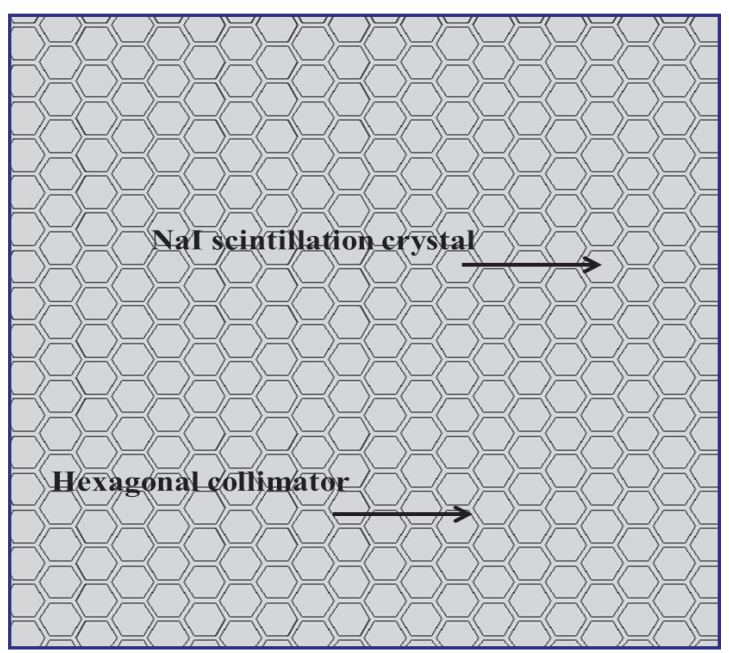

(b) Hexagonal hole collimator

Fig. (3): GATE visualization of the SPECT system.

\section{Lead Collimator}

One of the most important parts of a SPECT head is the collimator. Knowing its exact structure is essential to achieve more accurate MC simulation results (Assie et al., 2004). The specifications of Symbia E were used for the LEHR, LEGP and LEHS collimators to model them within the GATE simulation. Distances between the air-holes are calculated as the following (Fig. 4):

$\Delta \mathrm{y}=2 \mathrm{r}+\mathrm{s}$

$\Delta \mathrm{z}=2(\sqrt{3} \mathrm{r}+\mathrm{s} \cos 30)$

where $\Delta y, \Delta z$ are the distances between the centers of two consecutive holes along the $\mathrm{Y}$ and $\mathrm{Z}$ axes ,$t=d / \sqrt{ } 3$ is the hole radius (hexagon side), $d=2 r$ is the hole diameter between the flats, $s$ is the septal thickness and $f=s \cos \left(30^{\circ}\right)$. The total number of hexagon patterns is given by:

$n_{t}=2 n_{y} n_{z}$,
$\left(n_{y}-1\right) \Delta y=y$

$$
\left(n_{z}-1\right) \Delta z=z
$$

where $n_{y}$ is the number of hexagonal patterns along the Y-axis, $n_{z}$ is the number of hexagonal patterns along the Z-axis, $y$ and $z$ are the overall dimensions of the collimator.

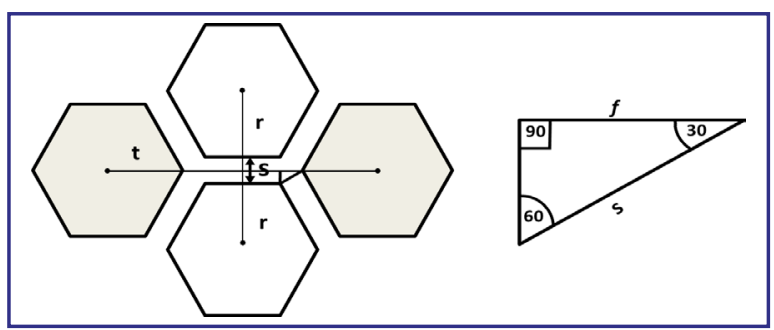

Fig. (4): The collimator geometry (the small triangle on the left is expanded on the right).

\section{Quadrant Bar Phantom}

The QB phantom (VICTOREEN 76-815) is a test tool which provides a simple and effective way of checking the scintillation camera's intrinsic spatial resolution. It is consisting of lead bars of varying widths and separations. The phantom was simulated by defining its overall dimensions $46 \times 46 \times 1 \mathrm{~cm}^{3}$ with 
plastic outer frame and internal lead bars within the GATE application. In this phantom, the lead is or-

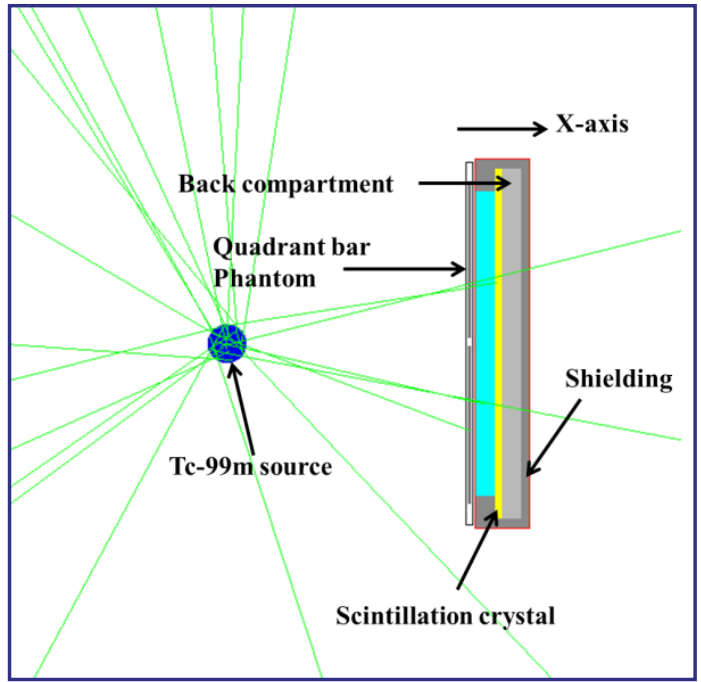

(a) Side view ganized into four groups of parallel bars with different widths as shown in Fig. 5.

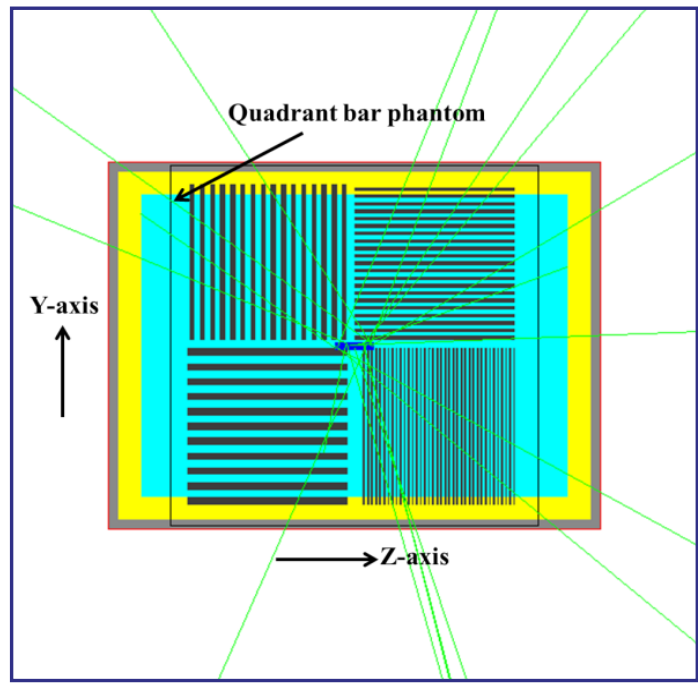

(b) Front view

Fig. (5): The visualization of the QB phantom.

\section{SPECT Quality Controls}

For SPECT QC, the intrinsic measurements are performed without the collimator and the extrinsic measurements are taken with the collimator installed. Some final calculations were performed by using the ImageJ (IJ) software with the International Atomic Energy Agency - Nuclear Medicine Quality Control (IAEA-NMQC) plugin (IAEA, 2017; Abdalrhman et al. 2017). This plugin is a set of Fiji (IJ upgrade) based codes developed in Java that allow to process and analyze the NM images acquired for the QC of gamma cameras and SPECT systems. NMQC software package can read the DICOM format and has the ability to read any other image formats that are supported by Fiji. In addition, another plugin, NucMed, was used to open and save the interfile images from the GATE simulations in IJ and Fiji. To analyze the simulation results, the ROOT analysis software was used to obtain the system spatial resolution values from GATE for different collimators and to calculate the FWHM along the $\mathrm{Y}$ and $\mathrm{Z}$ axes as well as studying the sensitivity dependence on the distance from the source for different collimators.

\section{Energy Resolution}

The energy resolution was determined for the gamma camera to measure the ability of the camera to distinguish between slightly different energies of the primary and scattered gamma radiation. It is a daily QC test that uses a radioactive source covering the full field of view, and it can be done extrinsically or intrinsically. The camera should be calibrated for energy (peaked off) before it can be used clinically. It is conventionally quantified as the FWHM of the photopeak divided by the photo-peak energy, $E_{p}(140 \mathrm{keV}$ for Tc-99m). This can be expressed as:

$$
\text { Energy Resolution }=\frac{\mathrm{FWHM}}{E_{P}} \times 100 \% .
$$

\section{Spatial Resolution}

Spatial resolution can be evaluated extrinsically and intrinsically. It is the ability of the camera to distinguish between two point or thin line sources of radiation to accurately detect the original location of a gamma ray in the Y-Z plane. It should be carried out weekly in clinical practice. 
Intrinsic spatial resolution can be calculated by the Modulation Transfer Function (MTF) in Fiji. The MTF is the ability of a system to reproduce a source containing an object of varying contrast, and presented as a graph of modulation $(0 \leq \mathrm{MTF} \leq 1)$ versus spatial frequency. The spatial frequency is measured as line pairs per $\mathrm{mm}(\mathrm{lp} / \mathrm{mm})$ and demonstrates how a pair of lines (lead and plastic bars) is like crests and peaks of a wave. If the MTF is approximately one, it indicates that the imaging modality have a very good spatial resolution $(\sim 100 \%)$. The percentage contrast is typically defined as:

$$
C=\frac{I_{\mathrm{MAX}}-I_{\mathrm{MIN}}}{I_{\mathrm{MAX}}+I_{\mathrm{MIN}}} \times 100 \%,
$$

where $I_{\mathrm{MAX}}$ is the maximum intensity and $I_{\mathrm{MIN}}$ is the minimum intensity of the source object or the resulting image. The MTF value can then be calculated by:

$$
\mathrm{MTF}=\frac{C_{\mathrm{IMG}}}{C_{\mathrm{OBJ}}},
$$

where $C_{\mathrm{IMG}}$ is the contrast of the resulting image from the SPECT system, and $C_{\mathrm{OBJ}}$ is the contrast of the original object (usually considered as 100\%).

The system extrinsic spatial resolution was simulated by using a small Tc-99m point source $1 \mathrm{~mm}$ in diameter placed at different distances $(5,10,15,20$ and $50 \mathrm{~cm}$ ) from the surface of the different collimators, LEHR, LEGP and LEHS by using a $\pm 20 \%$ energy window. It is affected by the collimator type and degrades as the distance from the radiation source to the collimator surface increases. In addition, the dependence of the extrinsic sensitivity on the source distance for each collimator was also estimated.

\section{Image Uniformity}

The uniformity is the QC procedure which checks that the camera response has the ability to produce uniform images. It should be performed daily for SPECT systems to ensure the quality of the resulting images. It can vary for the different radionuclides and the as- sociated energy windows. The uniformity measurements can be performed intrinsically or extrinsically.

Two uniformity parameters are computed for intrinsic measurements, Integral Uniformity (IU) and Differential Uniformity (DU). IU is measured over the Full Field Of View (FFOV) region of the SPECT camera which covers the entire exposed crystal surface, expressed as follows:

$\mathrm{IU}=\frac{N_{\mathrm{MAX}}-N_{\mathrm{MIN}}}{N_{\mathrm{MAX}}+N_{\mathrm{MIN}}} \times 100 \%$,

where $N_{\max }$ is the maximum count and $N_{\min }$ is the minimum count found in any pixel over the whole region of interest. The DU is defined as the IU except that the contrast is measured in a very small region. This measurement is usually performed using all the $5 \times 1$ pixel areas along the $\mathrm{Y}$ and $\mathrm{Z}$ axes and the maximum value is determined as:

$$
\mathrm{DU}=\max \left(\frac{N_{\max }-N_{\min }}{N_{\max }+N_{\min }}\right) \times 100 \% .
$$

The integral and differential values were measured by the equipment directly and also calculated by Fiji. Both can be determined for the Useful Field Of View (UFOV) and the Central Field Of View (CFOV). The UFOV is $95 \%$ of the FFOV, and CFOV is $75 \%$ of the UFOV. Fig. 6 shows the definition of the FFOV, UFOV and CFOV regions.

\section{Sensitivity}

The sensitivity of the SPECT system is determined by taking the ratio of the count for the detected photons within the energy window per minute per unit activity of the radioactive source in micro-Curies $(\mathrm{cpm} / \mu \mathrm{Ci})$. The standard sensitivity is calculated for LEHR by using a Petri dish which is a circular flat bottomed plastic container $10 \mathrm{~cm}$ in diameter and $1 \mathrm{~cm}$ in height filled with Tc-99m solution and positioned at some distance from the surface of collimator (see Fig. 7). The simulated sensitivity was obtained by modeling the dish at $10 \mathrm{~cm}$ from the LEHR collimator surface with an average deadtime of $1 \mu \mathrm{s}$. 


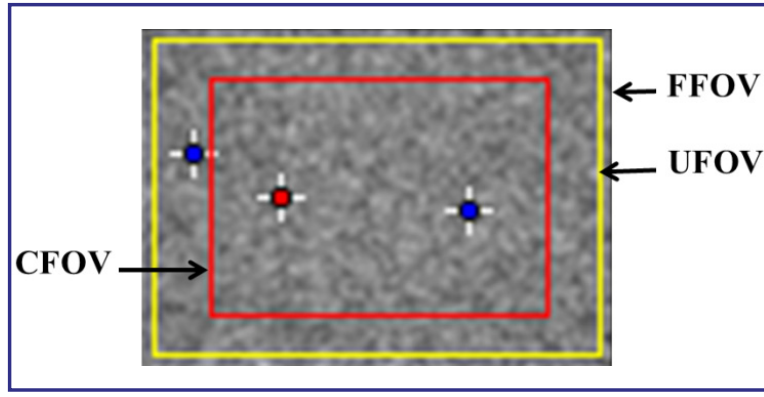

Fig. (6): The FFOV, UFOV and CFOV regions.

\section{RESULTS AND DISCUSSIONS}

\section{Energy Resolution}

As shown in Fig. 8a, the simulation results could be used to calculate not only the primary (un-scattered) photons but also the scattered photons in the crystal of the SPECT camera which is extremely dif-

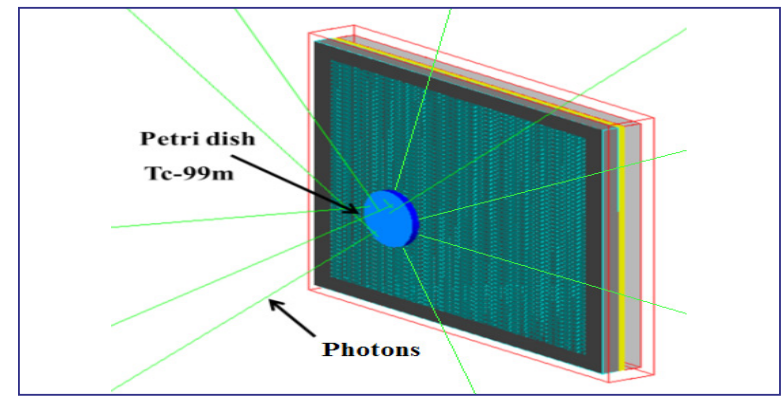

Fig. (7): Simulated Petri dish for sensitivity.

ficult to evaluate experimentally. The energy spectrum of a Tc-99m source was analyzed by the ROOT software. Measured and simulated energy spectra were compared with each other as indicated in Fig. 8 b. The FWHM and energy resolution were evaluated for the measured and simulated cases as listed in Table 2.

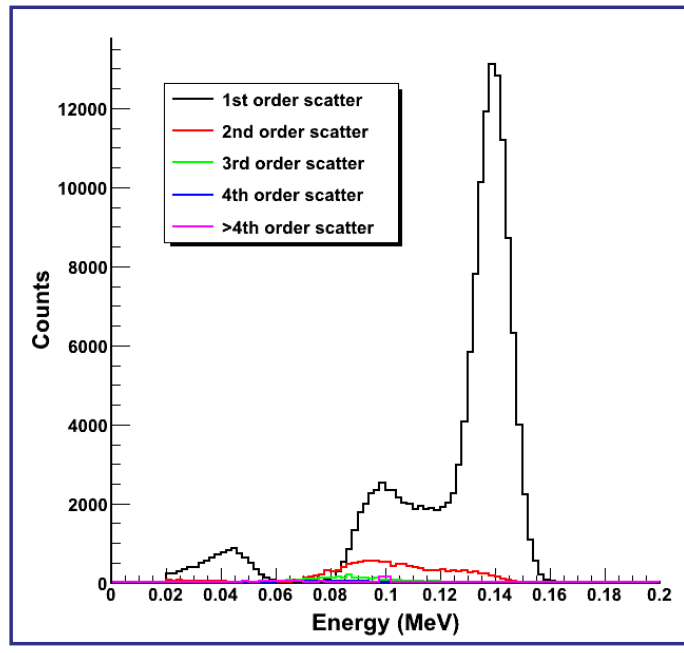

(a) Scatter orders

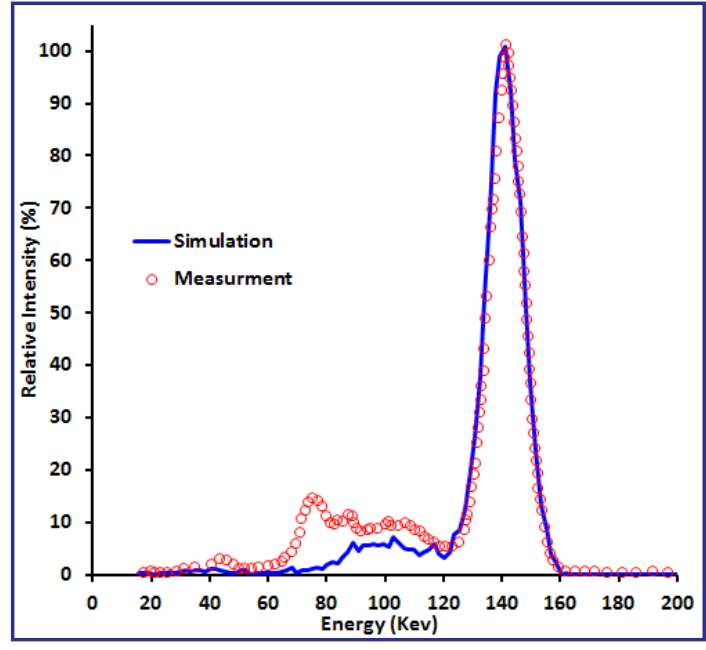

(b) Energy spectra

Fig. (8): Measured and simulated photon energies in the crystal for Tc-99m.

Table (2) : Photo-peak FWHM and energy resolution for Tc-99m.

\begin{tabular}{|c|c|c|}
\cline { 2 - 3 } \multicolumn{1}{c|}{} & FWHM (keV) & Energy Resolution (\%) \\
\hline Measurement & 12.82 & 9.16 \\
\hline Simulation & 13.20 & 9.43 \\
\hline Percentage Error & $\sim 3 \%$ & $\sim 3 \%$ \\
\hline
\end{tabular}




\section{Spatial Resolution}

The measured and simulated images for the intrinsic spatial resolution of the QB phantom are shown in Fig. 9. All the MTF results listed in Table 3 indicate that the simulated images are more resolved than the experimental images, especially for

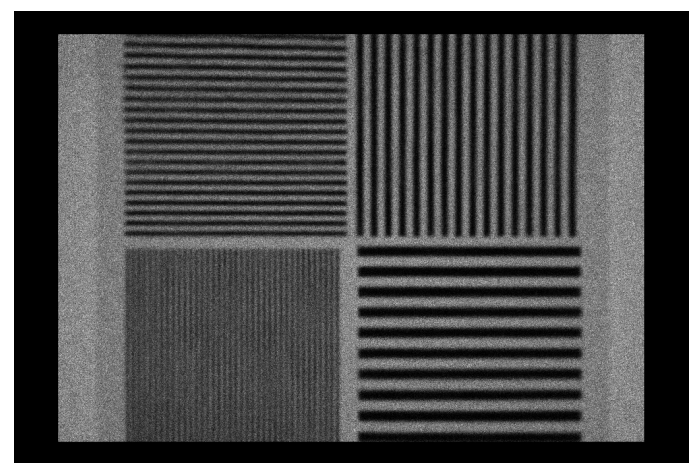

(a) Measurement the small bars. The crucial experimental factors that degrade the actual intrinsic spatial resolution are the electronic noise, component failures, and the poor alignment of the PMTs. These factors were not taken into consideration during the simulation stage. Fig. 10 shows the measured and simulated MTF curves.

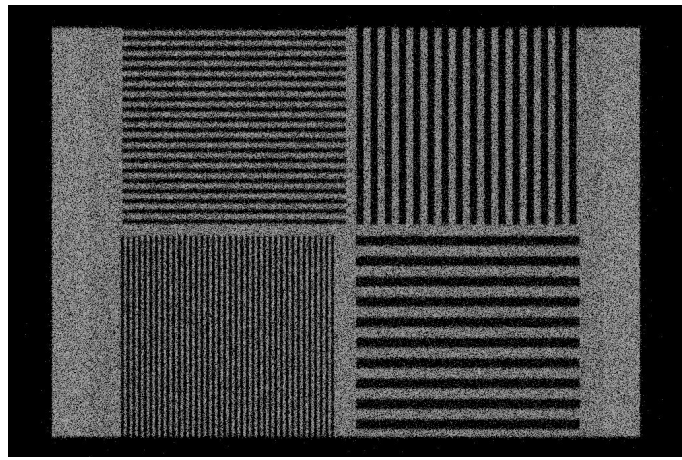

(b) Simulation

Fig. (9): The measured and simulated QB phantom images.

Table (3): Measured and Simulated MTF values.

\begin{tabular}{|c|c|c|c|c|c|c|c|c|}
\hline \multirow{2}{*}{$\begin{array}{c}\text { Bar width } \\
(\mathbf{m m})\end{array}$} & \multicolumn{9}{|c|}{ Angle of the QB phantom } \\
\cline { 2 - 9 } & \multicolumn{9}{|c|}{ Measurement } & \multicolumn{4}{c|}{ Simulation } \\
\cline { 2 - 9 } & $\mathbf{0}^{\circ}$ & $\mathbf{9 0}^{\circ}$ & $\mathbf{1 8 0}^{\circ}$ & $\mathbf{2 7 0}^{\circ}$ & $\mathbf{0}^{\circ}$ & $\mathbf{9 0}^{\circ}$ & $\mathbf{1 8 0}^{\circ}$ & $\mathbf{2 7 0}^{\circ}$ \\
\hline $\mathbf{9 . 5 2 5}$ & 0.93 & 0.96 & 0.94 & 0.94 & 0.89 & 0.90 & 0.88 & 0.90 \\
\hline $\mathbf{6 . 3 5 0}$ & 0.77 & 0.78 & 0.74 & 0.77 & 0.88 & 0.88 & 0.87 & 0.88 \\
\hline $\mathbf{4 . 7 6 3}$ & 0.56 & 0.57 & 0.60 & 0.60 & 0.85 & 0.85 & 0.85 & 0.85 \\
\hline $\mathbf{2 . 5 4 0}$ & 0.17 & 0.17 & 0.15 & 0.13 & 0.83 & 0.76 & 0.83 & 0.76 \\
\hline
\end{tabular}

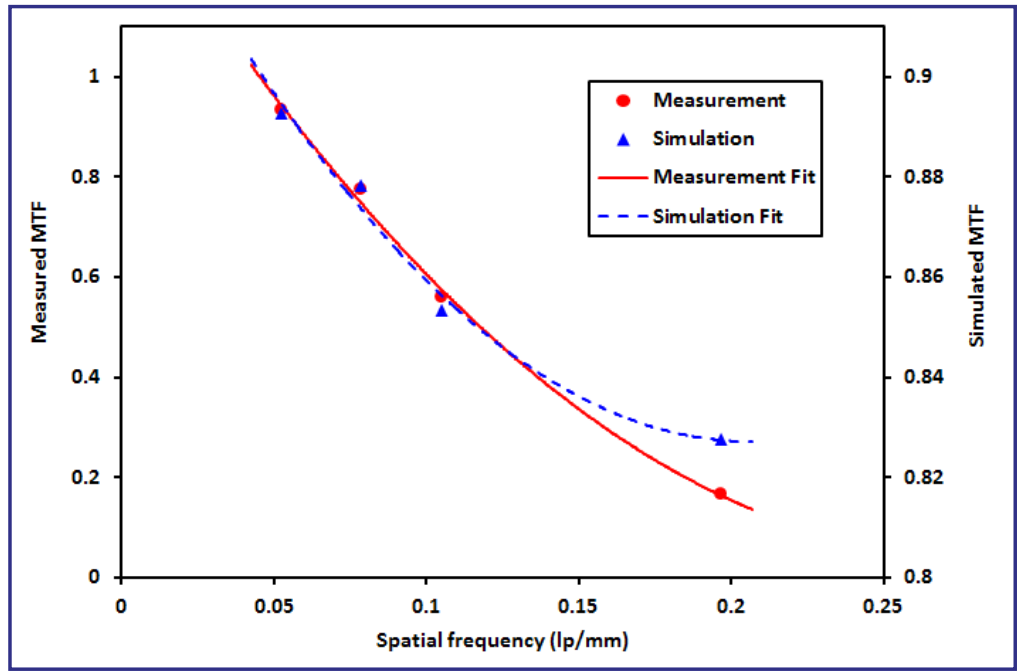

Fig. (10): Comparison between the measured and simulated MTF values. 
Fig. 11 shows the Gaussian fits used to calculate the FWHMs for the LEHR collimator at $5 \mathrm{~cm}$ along the $\mathrm{Y}$ and $\mathrm{Z}$ axes in ROOT. Based on the simulation results, the system spatial resolution increases with the increase of the septal thickness and degrades as the distance from the radiation source to the collimator surface increases, so that the LEHR collimator achieves the highest spatial resolution then the LEGP and LEHS collimators respectively as demon-

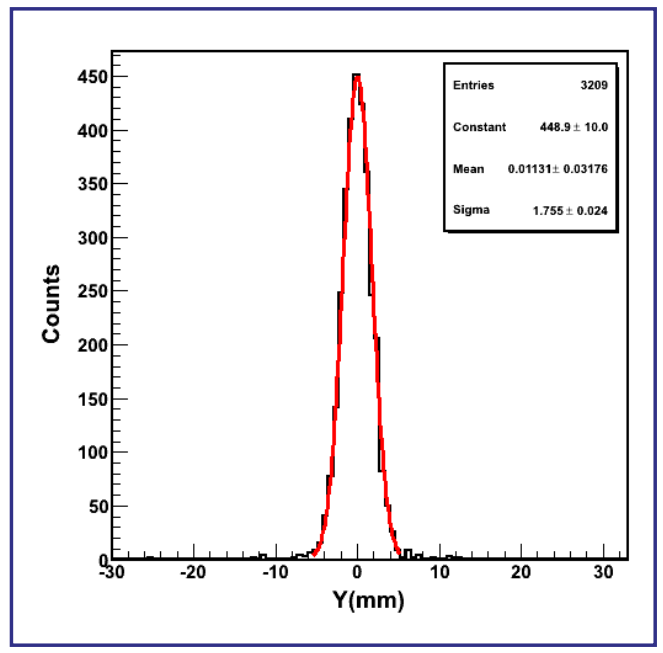

(a) Distribution of photons along the Y-axis strated in Fig. 12a. On the other hand, Fig.12b studies the sensitivity of the different collimators at different distances. The results show that the sensitivity decreases with the decrease of the spetal thickness, therefore the LEHR collimator has a less sensitivity than the other collimators and the LEHS achieves the highest sensitivity with very little dependence on the distance.

Fig. (11): The FWHM calculations for LEHR at $5 \mathrm{~cm}$.

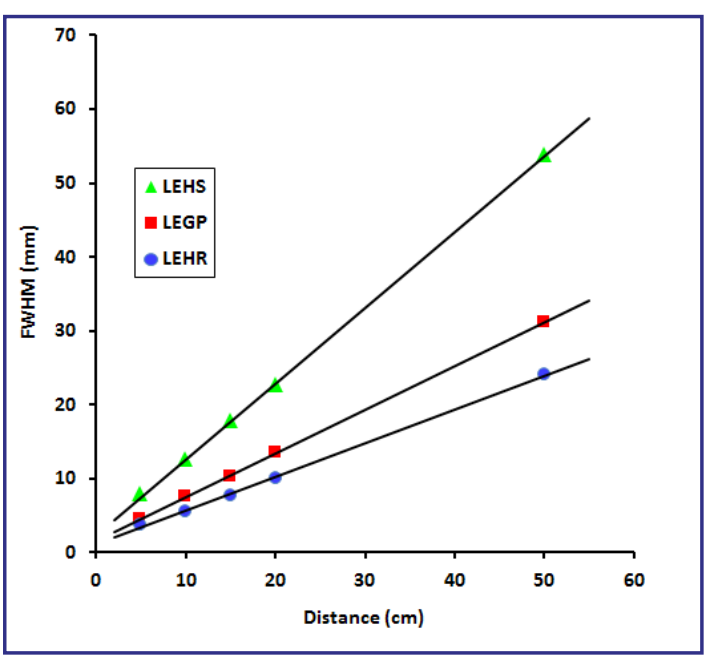

(a) Spatial Resolution

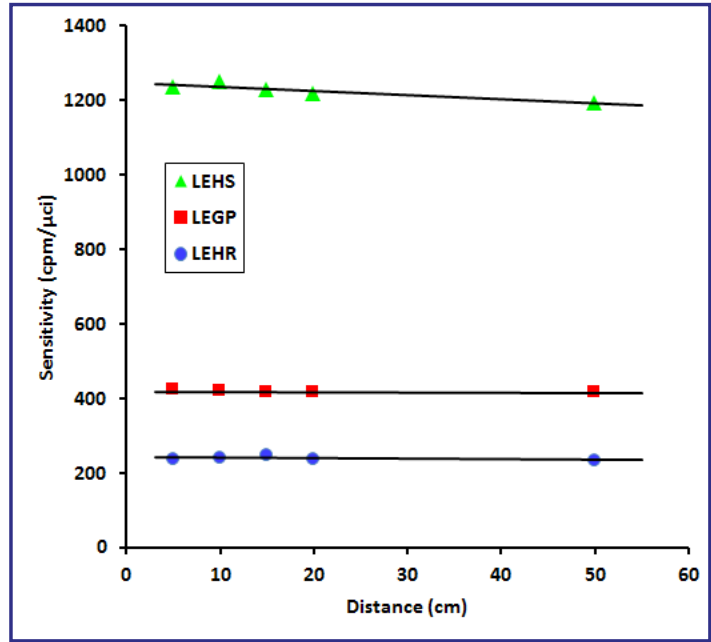

(b) Sensitivity

Fig. (12): The simulated system parameters for different collimators. 


\section{Uniformity}

The IU and DU results for both the UFOV and CFOV regions are shown in Fig. 13 and listed in Tables 4 and 5 . The calculated uniformity values in Fiji are close to the directly measured values by the equipment itself. But the simulated IU values are

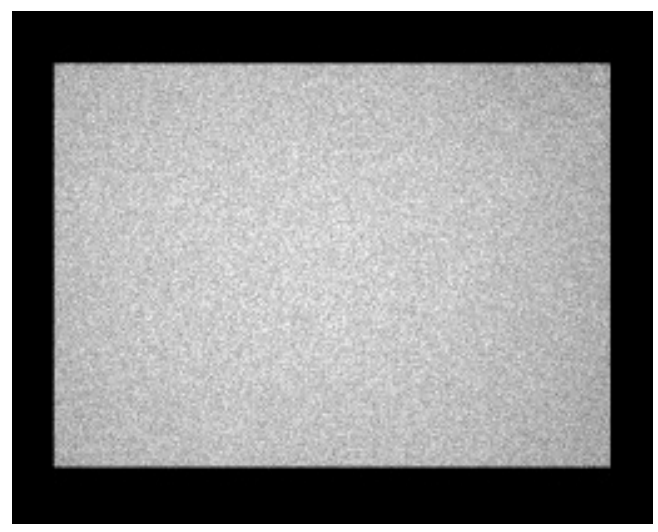

(a) Measurement smaller (better) as expected due to the actual degrading experimental factors which were not included in the simulation. On the contrary, the simulated DU is larger than the measured values due to the limitation on the simulation events.

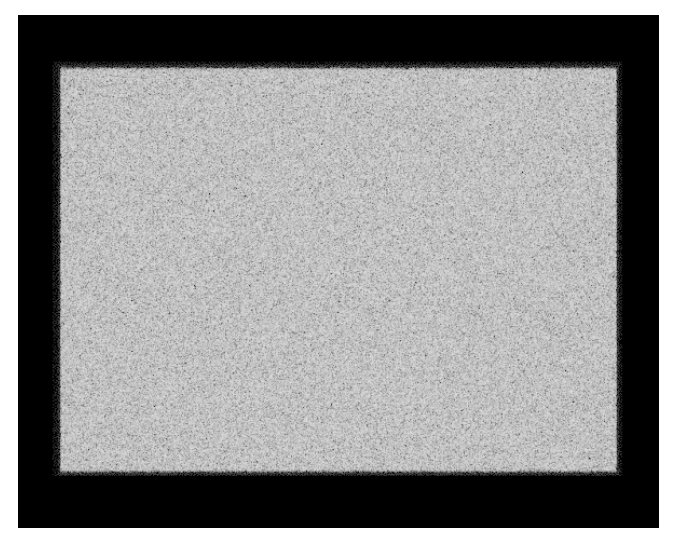

(b) Simulation

Fig. (13): The measured and simulated uniformity.

Table (4) : Measured and calculated intrinsic uniformity.

\begin{tabular}{|c|c|c|c|c|c|c|}
\cline { 2 - 7 } \multicolumn{1}{c|}{} & \multicolumn{3}{c|}{ UFOV } & \multicolumn{3}{c|}{ CFOV } \\
\hline Uniformity (\%) & Measured & Calculated & Difference & Measured & Calculated & Difference \\
\hline Integral & 12.98 & 13.43 & +0.45 & 10.22 & 9.04 & -1.18 \\
\hline Differential & 3.81 & 4.48 & +0.67 & 3.18 & 3.86 & +0.68 \\
\hline
\end{tabular}

Table (5) : Simulated uniformity.

\begin{tabular}{|c|c|c|}
\hline Uniformity (\%) & UFOV & CFOV \\
\hline Integral & 8.10 & 7.77 \\
\hline Differential & 6.46 & 6.46 \\
\hline
\end{tabular}

\section{SENSITIVITY}

The nominal datasheet sensitivity of the LEHR collimator for Symbia E is $202 \mathrm{cpm} / \mu \mathrm{Ci}$, while the simulated sensitivity result was $249 \mathrm{cpm} / \mu \mathrm{Ci}$. The higher sensitivity value obtained by the simulation is due to the fact that the implemented gamma camera model obviously did not take into account the real crystal quantum efficiency, small gaps (dead spaces) between the PMTs, deadtime mismatch, and experimental signal overlap present in the actual system.

\section{CONCLUSIONS}

The field of nuclear medicine is characterized by the use of open radioactive sources for the diagnosis and therapy of patients. Hence, the quality control procedures in such an environment may not 
be considered a luxury at all. In the current work, the analysis of SPECT images acquired for QC tests was performed by using the IAEA-NMQC plugins in Fiji. The design, modeling, and simulation of the SPECT modality were carried out in the framework of the Geant4/GATE application. Comparisons between the simulation results and the measurements indicated several consistencies within the limitations of the implemented models. Adding the missing experimental factors such as the electronic noises, PMTs gains, accurate deadtimes, etc. to the simulation models could have improved the match with the measurements but it would require more elaborate technical analysis during the maintenance time. This proposed research extension will be the subject of another future work by the authors.

\section{REFERENCES}

- Abdalrhman, N.E.; Maria, R.F.; Mario, D. et al. (2017): Quality control in dual head $\gamma$-cameras: Comparison between methods and software used for image analysis, ISSSD Proceedings, Santo Domingo, Dominican Republic.

- $\quad$ Agostinelli, S.; Allison, J.; Amako, K. et al. (2003): GEANT4-A simulation toolkit, Nucl. Inst. \& Meth. in Phys. Res. A 506, 250-303.

- Assie, K.; Breton V.; Buvat, I. et al. (2004): Monte Carlo simulation in PET and SPECT instrumentation using GATE, Nucl. Inst. \& Meth. in Phys. Res. A 527, 180-189.
- Buvat, I.; Castiglioni, I. (2002): Monte Carlo simulations in SPET and PET, Q. J. Nucl. Med. 46, 48-61.

- IAEA (2009): Quality Assurance for SPECT Systems, IAEA Human Health Series No. 6. IAEA, Vienna.

- IAEA (2017): IAEA-NMQC Toolkit for Fiji Application, Version 1.00, User's Manual, IAEA, Vienna.

- Jan, S.; Santin, G.; Strul, D. et al. (2004): GATE: a simulation toolkit for PET and SPECT, Phys. Med. Biol. 49, 4543-4561.

- $\quad$ Momennezhad, M; Sadeghi, R.; Nasseri, S. (2012): Development of GATE Monte Carlo simulation for a dual-head gamma camera, Radio. Phys. \& Tech. 5(2), 222-228.

- $\quad$ Park, M.J.; Park, K.S.; Leek, J.-S.; Kim, Y.-K. (2009): Validation of a GATE Model for the Simulation of a Trionix TRIAD SPECT Camera, J. Kor. Phys. Soc. 55(2), 681-687.

- $\quad$ SIEMENS (2014): SIEMENS Symbia E and S system specifications, SIEMENS MI-1263.

- Taherparvar, P.; Sadremomtaz, A. (2018): Development of GATE Monte Carlo simulation for a CsI pixelated gamma camera dedicated to high resolution animal SPECT, Aust. Phys. Eng. Sci. Med. 41(1), 3139.

- Zaidi, H. (1999): Relevance of accurate Monte Carlo modeling in nuclear medical imaging, Med. Phys. 26, 574-608. 